http://dx.doi.org/10.35381/r.k.v5i4.970

\title{
Retos e implicaciones de la aplicación del lenguaje XBRL en la economía popular y solidaria
}

\section{Rare and implied application of XBRL in the economy and solidarity}

\author{
Edison Roberto Noblecilla-Ortiz \\ edison.noblecilla05@esta.ucacue.edu.ec \\ Universidad Católica de Cuenca, Cuenca \\ Ecuador \\ https://orcid.org/0000-0002-3617-2053 \\ Edin Rodrigo Enriquez-Jaramillo \\ eenriquez@ucacue.edu.ec \\ Universidad Católica de Cuenca, Cuenca \\ Ecuador \\ https://orcid.org/0000-0001-8729-4205 \\ Verónica Paulina Moreno-Narváez \\ veronica.moreno@ucacue.edu.ec \\ Universidad Católica de Cuenca, Cuenca \\ Ecuador \\ https://orcid.org/0000-0003-1517-6124
}

Recibido: 01 de junio de 2020

Revisado: 20 de junio de 2020

Aprobado: 02 de septiembre de 2020

Publicado: 24 de septiembre de 2020 


\title{
RESUMEN
}

El objetivo fue diseñar un proyecto de desarrollo de la taxonomía XBRL en las empresas productoras del sector no financiero de la economía popular y solidaria para la edificación de una estructura de costos y determinación de precios justos conforme a lo dispuesto por la Ley Orgánica de Regulación y Control del Poder de Mercado. De tipo descriptiva. Los resultados reflejaron que en el $41 \%$ de empresas consultadas emiten estados financieros apoyados en un nivel alto por las herramientas tecnológicas, y tan solo un $13 \%$ mencionan que no cuentan con herramientas tecnológicas para la generación de estados financieros. El lenguaje XBRL permitirá a las cooperativas, asociaciones y comunitarios del sector no financiero popular y solidario etiquetar datos financieros para procesar y compartir datos de manera eficiente, de esta forma se logrará generar información uniforme relacionada con los costos de producción y determinación de precios justos.

Descriptores: Empresa; contabilidad; inventario; tributación. (Palabras tomadas del Tesauro UNESCO).

\begin{abstract}
The objective was to design a project for the development of the XBRL taxonomy in the companies that produce the non-financial sector of the popular and solidarity economy for the construction of a cost structure and determination of fair prices in accordance with the provisions of the Organic Law of Regulation and Control of Market Power. Descriptive type. The results reflected that $41 \%$ of the companies consulted issued financial statements supported at a high level by technological tools, and only $13 \%$ mentioned that they do not have technological tools to generate financial statements. The XBRL language will allow cooperatives, associations and communities of the popular and solidarity nonfinancial sector to label financial data to process and share data efficiently, in this way it will be possible to generate uniform information related to production costs and determination of fair prices.
\end{abstract}

Descriptors: Enterprises; accounting; inventories; taxation. (Words taken from the UNESCO Thesaurus). 


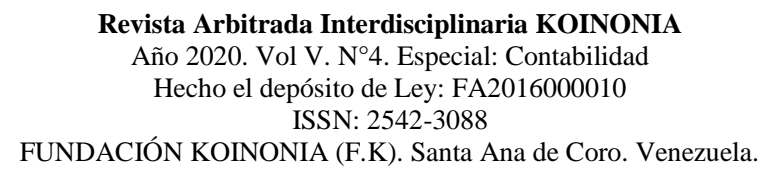

Edison Roberto Noblecilla-Ortiz; Edin Rodrigo Enriquez-Jaramillo; Verónica Paulina Moreno-Narváez

\section{INTRODUCCIÓN}

Por consiguiente, el XBRL es el acrónimo de Extensible Business Reporting Languaje, es un lenguaje de mercado creado en el año 1998 por el auditor y contable Charles Hoffman, para el intercambio electrónico de informes de negocios, a diferencia de otros formatos es destacable que XBRL es un lenguaje estándar abierto, su software es de uso libre y aplica a todo tipo de empresa (The Bussines Reporting Lenguage, 2020).

EI XBRL es un lenguaje que busca la transparencia financiera a nivel mundial, y se ha impuesto principalmente en sectores claves como aseguradoras, entidades financieras y empresas en general, en este sentido, se resalta la importancia de incorporar este lenguaje en las pequeñas empresas productoras que conforman la economía popular y solidaria.

Al respecto, se debe mencionar que la (Superintendencia de Economía Popular y Solidaria, 2019) define a la economía popular y solidaria como la organización económica en la que sus integrantes organizan y desarrollan procesos relacionados con la producción, comercialización, distribución, financiamiento y consumo de bienes y servicios, bajo un enfoque individual o colectivo, con la finalidad de satisfacer las necesidades de la población. Entre los principios de esta forma de organización, resaltan los siguientes:

1. Comercio justo, consumo ético y responsable.

2. Distribución equitativa y solidaria de excedentes.

3. Responsabilidad social y ambiental.

4. Rendición de cuentas.

5. Autogestión.

6. Solidaridad.

7. Búsqueda del buen vivir

8. Equidad de género, entre otros. 


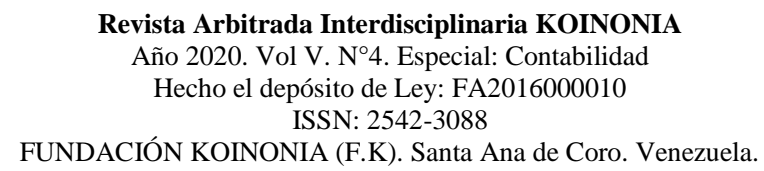

Edison Roberto Noblecilla-Ortiz; Edin Rodrigo Enriquez-Jaramillo; Verónica Paulina Moreno-Narváez

En cuanto a sus formas de organización, el sector popular y solidario está integrado por cooperativas, asociaciones y comunitarios regidos por la Ley de Economía Popular y Solidaria. En lo que respecta a las organizaciones económicas del sector asociativo, estas deben constituirse según la ley, al menos, por cinco personas naturales, consideradas productores independientes ya sea de bienes y servicios similares o complementarios, a fin de abastecer a sus asociados de insumos, materia prima, equipos , herramientas, tecnologías, o comercializar de manera conjunta su producción, mejorando a sí su capacidad competitiva. Por su parte, las cooperativas de producción se constituirán para producir y comercializar bienes o servicios de cualquier tipo, en este contexto, la economía popular y solidaria adquiere relevancia, ya que genera fuentes de empleo, soluciona problemas de las comunidades, dinamiza el emprendimiento, crean redes solidarias y económicas y aporta con divisas al país a través de la exportación de sus productos.

A pesar del rol fundamental que desempeñan estas organizaciones en la economía del Ecuador, las mismas atraviesan grandes dificultades al enfrentarse a políticas y regulaciones que no están acorde a su realidad, pues los esfuerzos del Estado por reconocer a este sector en la Constitución de Montecristi y la promulgación de una ley particular no han sido suficientes, ya que se ha puesto en evidencia debilidades que amenazan su funcionamiento y permanencia en el mercado, tales como: la falta de gobernabilidad que conlleva a la inadecuada toma de decisiones y escasa participación de sus cooperados; alta competencia en el sector debido a la falta de articulación entre productores de similares bienes o servicios; elevados costos de producción, ausencia de economías de escala, deficiente calculo de costos y precios, prácticas poco éticas de los dirigentes, entre otras.

Cabe señalar que la falta de gobernanza no ha permitido que las cooperativas, asociaciones y comunitarios sean vistos como una buena alternativa empresarial, por lo que el paradigma de la economía popular y solidaria, en la actualidad se aleja de la protección de los derechos del consumidor y de la determinación de precios justos, ante 
esta situación, en la presente investigación se plantea el siguiente problema: ¿cómo establecer una estructura de costos que facilite la determinación de precios justos en las empresas de producción no financiero popular y solidario en correspondencia con la Ley Orgánica de Regulación y Poder de Mercado?. A partir de esta problemática se propone como objetivo diseñar un proyecto de desarrollo de la taxonomía XBRL en las empresas productoras del sector no financiero de la economía popular y solidaria para la edificación de una estructura de costos y determinación de precios justos conforme a lo dispuesto por la Ley Orgánica de Regulación y Control del Poder de Mercado.

\section{Referencial teórico}

\section{Obtención de reportes financieros bajo XBRL}

La Norma Internacional de Contabilidad (NIC) 1 establece los requerimientos generales para la presentación de los estados financieros y la guía para determinar su estructura y requisitos mínimos sobre su contenido, es así que, su objetivo consiste en establecer las bases para la presentación de los estados financieros con propósitos de información general, para comparar de manera interna y externa, por consiguiente, la finalidad de los estados financieros es una representación de la realidad y utilidad financiera de una empresa, con el fin de dar información acerca de la situación financiera, el rendimiento financiero y los flujos de efectivo. Los componentes de los estados financieros son los siguientes:

1. Balance

2. Estado de resultados

3. Cambios en el patrimonio

4. Estado de flujo de efectivo

5. Notas, en las que se incluirá un resumen de las políticas contables significativas y otras notas explicativas. 
En este contexto, la adopción de las NIIF genera una importante capacidad de gestión a partir de la presentación de informes en tiempo real, por tanto, existe razones de peso para considerar la convergencia NIIF/ XBRL la cual se profundiza más adelante, principalmente debido a que los procesos tradicionales de recolección y posterior construcción manual de reportes son técnicas obsoletas que hacen que la generación de informes de cuentas sea cada vez más dificultosa. Al respecto, el sistema XBRL permitirá unificar el tratamiento de la información en un solo formato con fines de adaptación a la evolución económica del presente siglo, de esta forma se eliminarán procesos desactualizados y se aplicarán buenas prácticas tecnológicas encaminadas a optimizar los procesos contables y financieros, en consecuencia la adopción de las NIIF deber ser paralela a la implementación de XBRL en los reportes financieros para el procesamiento y transmisión de la información (Leal, 2015).

Es importante resaltar que $X B R L$ se encuentra libre de licencias, no se paga por su uso y se puede trabajar en cualquier sistema operativo, ordenador o teléfono móvil. Entre los principales documentos o reportes que se puede obtener a partir de su aplicación están:

1. Estados financieros

2. Libros mayores

3. Asientos diarios

4. Informes de cartera de crédito

5. Informes sobre riesgos

6. Informes sobre costos

7. Declaraciones de impuestos

8. Informes de riesgos

9. Informes de seguros

10. Información comercial 


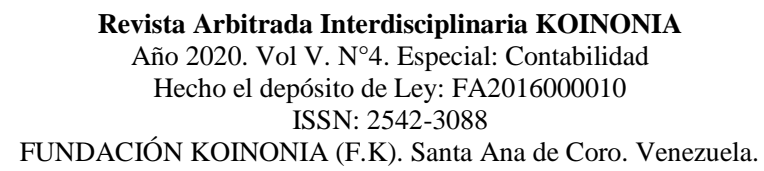

Edison Roberto Noblecilla-Ortiz; Edin Rodrigo Enriquez-Jaramillo; Verónica Paulina Moreno-Narváez

Entre las ventajas que incluye el XBRL según (Ponte, et al., 2007) y (Madrid, Cortés, \& Cardona, 2016), se menciona las siguientes:

1. Estandariza el proceso de codificación de tal forma que toda la información de un estado financiero específico puede ser importada a cualquier plataforma.

2. Posibilita la búsqueda rápida de información, ya que los datos se marcan con etiquetas que permiten que los datos relacionados se unifiquen en un solo informe.

3. Disminuye el riego de fallas en el ingreso de datos, ya que la información es digitada una sola vez.

4. Genera estados financieros generales incluido sus notas respectivas.

5. Permite rescatar a nivel de informes gerenciales información relevante, puesto que el sistema contable interactuar con la base de datos.

6. Facilita el reporte de impuestos en función de los formatos establecidos por la administración tributaria, además propicia el cruce de información y comparación de datos entre diferentes entidades a efectos de control y auditoría.

7. Permite la generación de informes bajo el marco de la metodología Scorecard .

8. Propicia rapidez en la presentación de la información con mayor transparencia, reducción de errores y costos.

9. Posibilita la creación de definiciones reutilizables y autorizadas, llamadas taxonomías; los reguladores encargados de establecer normas de contabilidad, las agencias gubernamentales y otros grupos desarrollan taxonomías que necesitan definir la información sobre la que se debe informar

Ante lo expuesto, (Zamarra, et al., 2017) precisan que el XBRL es un estándar internacional abierto para informes comerciales, administrado por un consorcio global sin fines de lucro de nombre XBRL Internacional, es adaptable a empresas del sector público como privado; patrocinado por el Instituto Americano de Contables Públicos Certificados (AICPA), su potencial a mas de asociarse a los sistemas contables permite la construcción de espacios específos mediante reportes corporativos que homogeneizan la demanda de información y permiten construir mayor diversidad. Cabe señalar que este 
lenguaje de programación no corresponde a un conjunto de reglas contables ni es traductor de los principios de contabilidad generalmente aceptados (PCGA), sus usos están encaminados a la generación de reportes internos y externos para la toma de decisiones (ver figura 1).

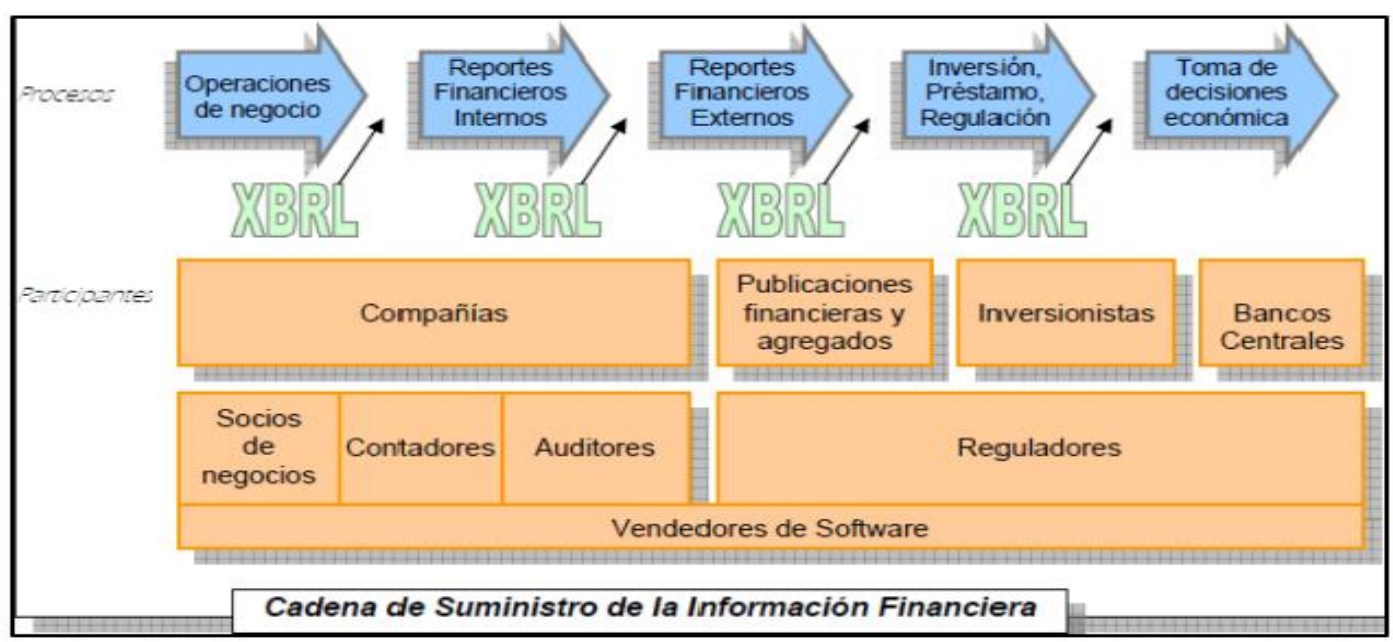

Figura 1. Cadena de suministro de la información financiera.

EI XBRL por sus características permite que la preparación de información sea rápida, sin embargo, para ello se requiere definir un modelo que representa los ítems que se va a comunicar mediante la creación de taxonomías. Estas taxonomías representan los diccionarios del lenguaje XBRL y consisten en esquema de clasificación mediante etiquetas que agrupan los siguientes elementos específicos de información:

Esquema: responde al conjunto de elementos que figuran en los informes y en la estructura de los mismos.

Linkbase de etiquetas: son etiquetas que se asocian a los elementos del diccionario, pueden configurarse en distintos idiomas, su propósito es construir las representaciones de los informes. 
Linkbase de referencias: se refiere a la base legal sobre la que se fundamenta el modelo, en este sentido, la normativa desempeña un rol importante al establecer los conceptos que se utilizaran para crear los informes.

Linkbase de presentación: corresponde a las reglas que se utilizaran en la representación del informe que se va a modelar.

Linkbase de cálculo: los parámetros de cálculo son operaciones matemáticas que forman parte de la taxonomía y su finalidad consiste en validar los informes XBRL.

Linkbase de definición: son reglas adicionales que posibilitan la documentación de relaciones entre elementos de la taxonomía a efectos de validar los informes.

Instancia XBRL: consiste en etiquetar cada valor del negocio para que pueda ser identificado con facilidad mediante una unidad de medida.

Informe XBRL: es el informe de negocio XBRL que incluye la taxonomía sobre la cual se desarrolló, la misma, permite validar los datos conforme a las definiciones, reglas, idiomas, entre otros.

Validador XBRL: es el procesador XBRL basado en XML que permite la validación de una instancia con reación a la taxonomía correspondiente

Visualizador XBRL: esta herramienta transforma una instancia XBRL a un fichero HTML de forma que sea visible en un navegador.

En cuanto a la implementación de $X B R L$ se requiere previamente definir la arquitectura tecnológica sobre la que se desarrollará, al respecto, se identificaran etiquetas específicas para cada elemento de información aprobadas por la XBRL International (ver figura 2). 


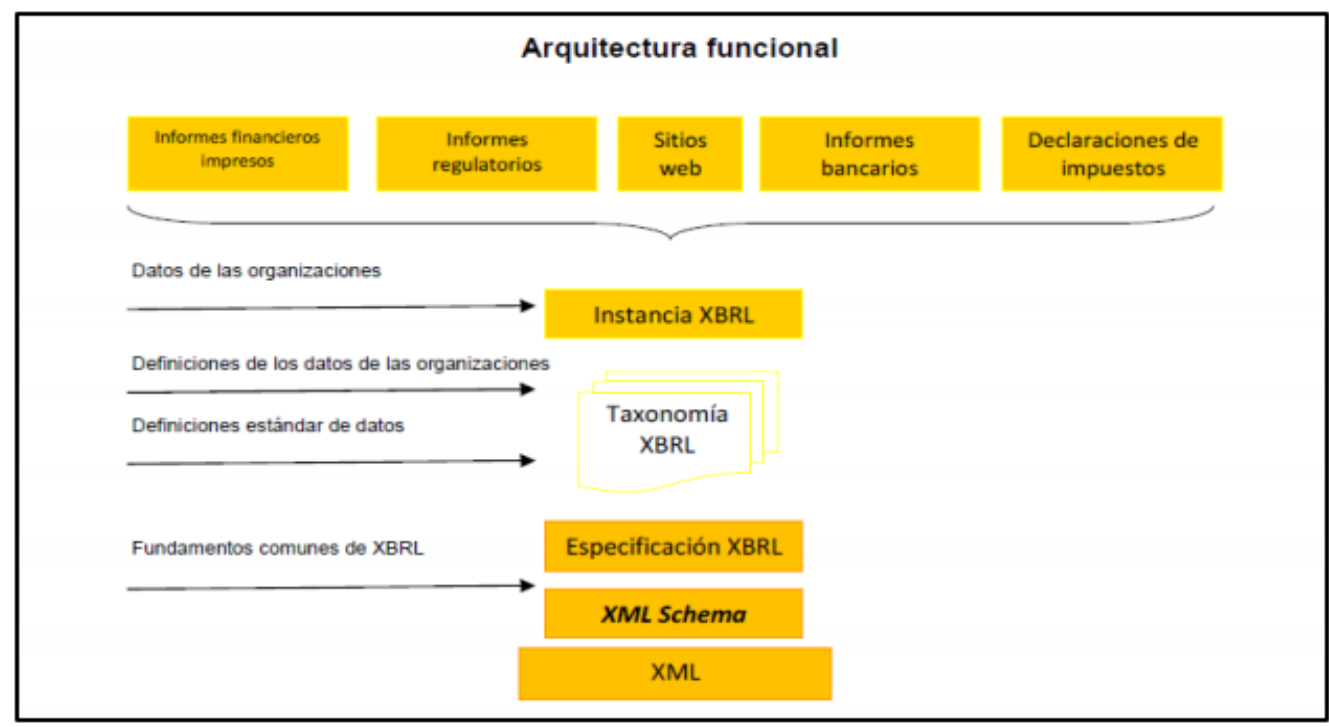

Figura 2. Arquitectura funcional de XBRL.

Fuente: Lucero (2017).

En lo posterior, se debe considerar el costo de implementación, que se encuentra asociado con los cambios tecnológicos que se deben emprender en las organizaciones. Ahora bien, considerando la experiencia española en cuanto a la implementación de XBRL, se puede definir la siguiente hoja de ruta a seguir por parte del sector empresarial:

1) Capacitar a las personas vinculadas con las áreas de sistemas, contabilidad y auditoría.

2) Adquirir las herramientas para la creación de taxonomías y validación de informes.

3) Crear las taxonomías XBRL con extensión a las taxonomías de IFRS o NIIF.

4) Distribuir la ayuda técnica entre varios profesionales para I generación de informes XBRL.

5) Crear por parte de los organismos de control las taxonomías y establecer la obligatoriedad de reportar la información en este formato de intercambio de información financiera. 


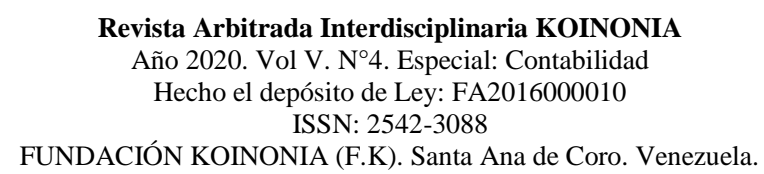

Edison Roberto Noblecilla-Ortiz; Edin Rodrigo Enriquez-Jaramillo; Verónica Paulina Moreno-Narváez

\section{La contabilidad de costos y su aporte a la toma de decisiones}

La contabilidad permite el manejo de la información y normalización de los resultados obtenidos por una empresa, por lo tanto, comprende los procedimientos de identificación, medición, registro y comunicación de la información económica, con la finalidad de que los gestores valoren sus resultados (Blacio-Aguilar, et al., 2020). Las ciencias contables se dividen en varias ramas como la contabilidad de gestión, la contabilidad ambiental, la contabilidad financiera, $y$, entre otras la contabilidad de costos, objeto de esta investigación.

En este contexto, la contabilidad de costos ofrece información real y concreta de todos los costos y gastos incurridos por las empresas en el almacenamiento y transformación de las materias primas en productos terminados, por consiguiente, sirve para establecer el costo de un producto y para tener control sobre la producción, la venta del producto, la administración y la financiación del mismo; el propósito es establecer las fases de almacenamiento y transformación de las materias primas, permitiendo conocer como se han utilizado los distintos factores productivos, analizar los costos y beneficios de los departamentos, actividades o productos de la empresa.

Por consiguiente, la contabilidad de costos se representa mediante un sistema de información que busca socializar información de tipo financiera y no financiera a la administración, con la finalidad de ejercer procesos de planificación, dirección, control y evaluación del desempeño organizacional. En efecto, como todo proceso la contabilidad de costos genera productos relevantes como son los estados financieros, que ha consideración de (Zapata, 2019) deben ser de calidad y oportunos a fin de justificar su presencia como fuente de información para la toma de decisiones. Además el autor precisa, que una empresa industrial debe preparar al menos los siguientes informes básicos:

1. Estado de costos de productos vendidos.

2. Estado de resultados integrales.

3. Estado de situación financiera. 


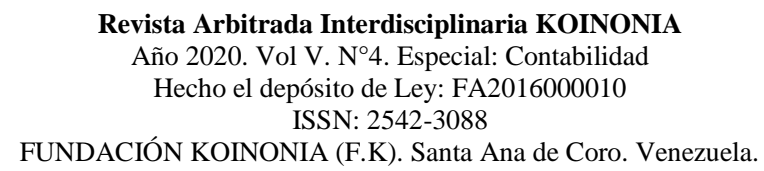

Edison Roberto Noblecilla-Ortiz; Edin Rodrigo Enriquez-Jaramillo; Verónica Paulina Moreno-Narváez

4. Estado de cambios en el patrimonio, y.

5. Estado de flujo de efectivo

En este orden de ideas, el costo de producción se entenderá como el valor monetario que se invierte en materia prima, mano de obra y gastos indirectos de fabricación requeridos para fabricar bienes o sus partes o generar servicios, en este sentido, a partir de esta definición se desprende el término elementos de producción los mismos que están vinculados entre sí y son necesarios dentro del proceso productivo. Al respecto, la materia prima se encuentra constituida por los bienes en estado natural o no requeridos para la fabricación de artículos; la mano de obra que corresponde a la creatividad del ser humano que puede ser física o intelectual y se utiliza para la transformación de la materia prima con la ayuda de equipos, herramientas, tecnologías, entre otros; e, insumos o gastos generales de fabricación que son aquellos bienes y servicios complementarios indispensables para generar un producto.

Por otro lado, los costos de producción tienen una clasificación convencional que pretende aclarar conceptos para su aplicación. Por su relación con el nivel de producción, los costos se estructuran en función del volumen de producción, en este sentido, los costos pueden clasificarse en fijos que son aquellos que permanece inalterables durante el proceso de la producción y variables son aquellos que crecen o decrecen en forma proporcional conforme aumente o disminuya la producción. Por el alcance, los costos pueden ser totales y representan la sumatoria de todas las inversiones realizadas en materia prima, mano de obra y gastos indirectos de fabricación para producir un lote o grupo de bienes; $y$, unitarios que corresponde a la división de los costos totales divididos para el número de unidades fabricadas. Por la identidad, los costos se puede agrupar en directos que son aquellos de fácil asignación a un producto o servicio; y, los indirectos que son aquellos que no se asignan con precisión y por tanto, requieren ser tratados como indirectos (Cárdenas-Pañi, et al., 2020). 


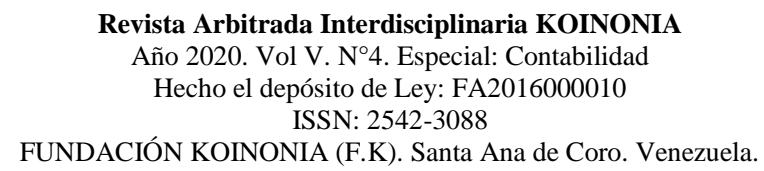

Edison Roberto Noblecilla-Ortiz; Edin Rodrigo Enriquez-Jaramillo; Verónica Paulina Moreno-Narváez

Por o expuesto, la estructura de costos es el resultado de un proceso orientado a organizar de manera práctica la gestión de costos, basado en las prioridades estratégicas y operativas de la organización; debe cubrir todas las operaciones y definir organismos para el procesamiento de datos financieros, para así desarrollar la capacidad de generación de información oportuna y de calidad a nivel interno y externo (Muñoz, et al., 2017).

\section{Retos e implicaciones del XBRL en Ecuador: base legal}

La Constitución del Ecuador expresa que el sistema económico, social y solidario se encuentra integrado por las formas de organización económica pública, privada, mixta, popular y solidaria. Al respecto, el país esta considerado como una de las naciones latinoamericanas que presenta los mayores avances en la economía popular y solidara, con un marco regulador vigente tanto para el sector financiero como para el no financiero que genera seguridad jurídica y ordena el accionar de sus actores, a su vez el cuerpo legislativo ha dado paso a una institucionalidad en diferentes instancias del Estado para el control, supervisión, regulación, financiamiento, fomento y diseño de políticas, tal es el caso de la creación de la Ley Orgánica de Regulación y Control de Poder de Mercado que a su vez permite la creación de la Superintendencia de Control del Poder de Mercado, su objetivo es alcanzar la eficiencia en los mercados, el comercio justo y el bienestar general de los consumidores.

En consecuencia, están sometidos a esta ley, todos los operadores económicos, sean personas naturales o jurídicas, públicas o privadas, nacionales o extranjeras, con o sin fines de lucro que ejecuten actividades económicas en todo o parte del territorio nacional, así como los gremios que las agrupen, y las que formalicen actividades económicas fuera del país que produzcan efectos perjudiciales en el mercado nacional (Morán, 2014). Retomado el sistema de economía popular y solidaria, este se encuentra integrado por organizaciones económicas individuales o colectivas creadas con la finalidad de desarrollar procesos de producción, intercambio, comercialización, financiamiento y 
consumo de bienes y servicios; sus relaciones de actuación se sustentan en principios de solidaridad, cooperación, reciprocidad, trabajo, buen vivir, además presentan características que las diferencias de las corporaciones o empresas privadas tal como se aprecia en la figura 3.

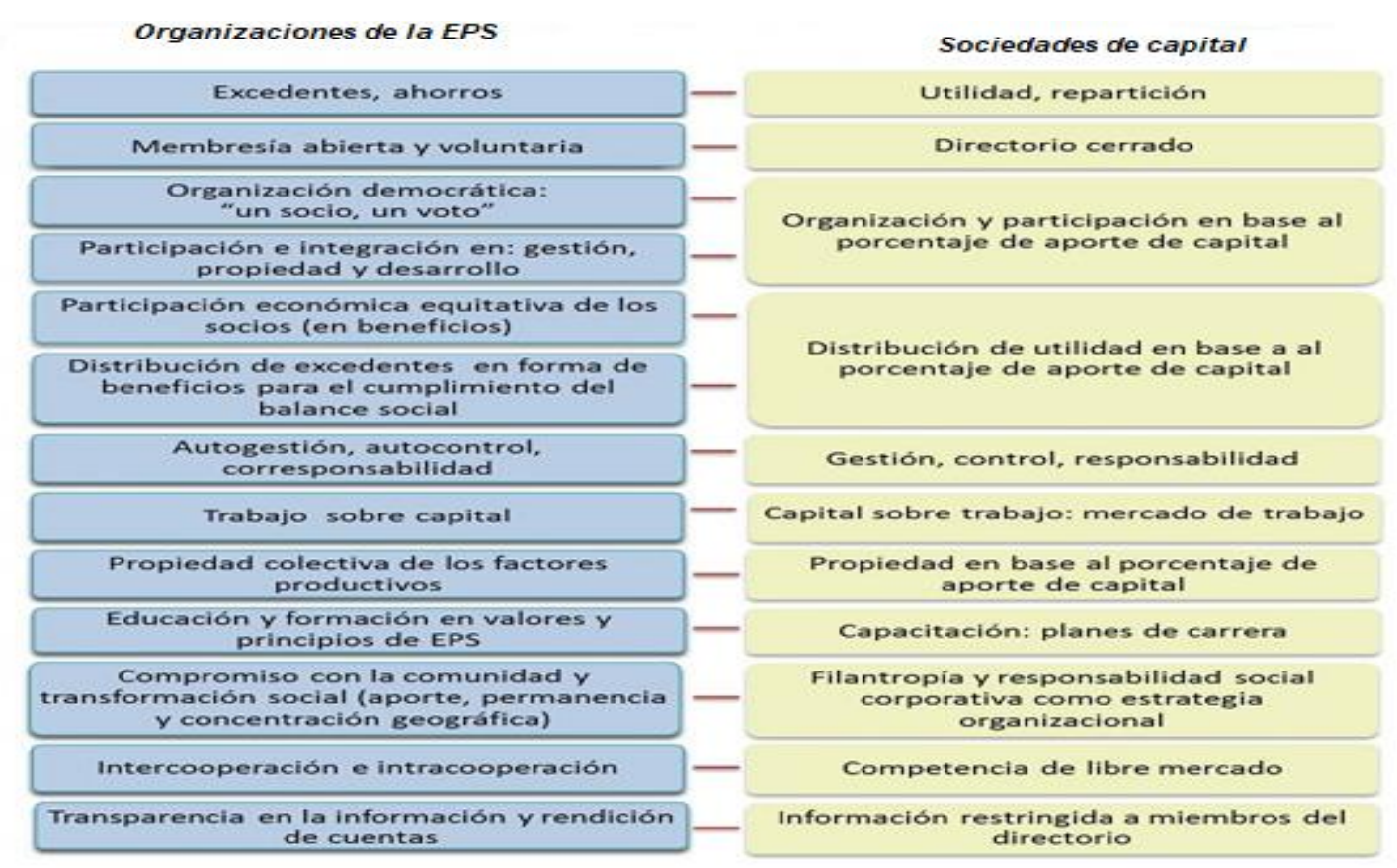

Figura 3. Características diferenciadoras entre la economía popular y solidaria y las sociedades de capital.

Fuente: Superintendencia de Economía Popular y Solidaria (2019).

La economía popular y solidaria se encuentra integrada por tres sectores: a) Asociaciones integradas por personas naturales dedicadas a actividades productivas similares 0 complementarias, su finalidad es comercializar sus productos de manera autogestionada y solidaria; b) Cooperativas comprendidas por sociedades de personas unidas de forma libre y voluntaria para ejercer actividades de producción, comercialización, servicios, vivienda, ahorro y crédito, y consumo; y, c) Comunitario integrado por organizaciones en función de territorio, familia, cultura, entre otros, que bajo la filosofía de trabajo conjunto, 
tienen como objetivo producir, comercializar, distribuir, y consumir bienes y servicios lícitos (Ibídem).

Este modelo económico se encuentra estrechamente ligado con la práctica de comercio justo y se fundamenta en los principios de solidaridad, predominio del trabajo sobre el capital, redistribución equitativa de los ingresos y fomento de la relación directa entre productores y consumidores para evitar la intermediación, en sí, esta práctica busca la generación de oportunidades para los productores en desventaja económica, persigue el pago de un precio justo sin desvalorizar el esfuerzo y trabajo de los productores, propende a la equidad de genero y generación de condiciones dignas de trabajo para los productores, por tanto, presenta una estructura que busca disminuir la concentración en pocas empresas y modificar las injustas relaciones que privilegian a las grandes economías mundiales. A pesar de la mínima participación de productores en los distintos países, se avizora una dinámica alentadora en cuanto a la tendencia creciente del comercio justo, considerado como una alternativa idónea para mejorar la calidad de vida de los pequeños productores. En la tabla 1 se presenta el resumen de las principales diferencias entre el comercio justo y el comercio convencional. 


\section{Tabla 1.}

Diferencias entre comercio justo y comercio convencional.

\section{Comercio justo}

Es un sector importante en la economía solidaria

Su fin último no es el lucro

Da oportunidades a los grupos productores desfavorecidos

Promueve el contacto directo entre comprador y vendedor

Apoyo y colaboración entre productor y comercializador

Fortalecimiento de capacidades

Asesoramiento técnico para el desarrollo de las personas y sus productos

Pedidos y pagos garantizados

\section{Comercio convencional}

Se inscribe en la economía capitalista

El fin último es la ganancia y la acumulación

No se contemplan las circunstancias y el desarrollo del productor

Solo importa el producto, ignorando las condiciones del productor

Ausencia de ganancia justa para el productor

Inexistencia de un proyecto social

Relaciones comerciales y continuidad no garantizadas

Solo es relevante que el producto sea enviado en conformidad con el acuerdo comercial

En el contexto de la práctica de precios justos, se requiere pagar al productor un precio justo que le permita alcanzar una vida digna, por ello, el precio no debe ser impuesto sino consensuado, debiendo cubrir los costos reales de producción y dotar de un margen que posibilite la producción sostenible, en este escenario, toma importancia la estructura de costos, ya que la misma permite la fijación de precios a partir del reconocimiento del nivel de costos como punto de partida, en este sentido, la fijación de precios se fundamenta en la estructura de costos de materias primas, mano de obra, costos indirectos de fabricación y la utilidad esperada, cabe resaltar que, en la legislación tributaria del Ecuador, se reconocen los costos y gastos de producción solo si cuentan con los respaldos de facturas emitidas por el vendedor (Ordóñez, et al., 2020).

A partir de lo expuesto, se deduce la importancia de contar con estructuras estandarizadas para la generación de información tanto para usuarios internos como para usuarios externos, ante ello, se debe considerar que la adopción de este lenguaje 
requiere de cambios sustanciales conforme los marcos legales que sean destinados para el despliegue en Ecuador. En el contexto de las experiencias obtenidas con la implementación en otros países, se considera importante el uso de la tecnología en las entidades de vigilancia y control del intercambio de información contable, financiera ya que esto promueve la transparencia de las operaciones comerciales y la rendición de cuentas a las partes involucradas (Lucero-Benítez \& Trujillo-Prada, 2018).

Por su parte, (Zamarra, et al., 2018), mencionan que la necesidad de formular y reglamentar el XBRL en el sector popular y solidario, surge del proceso de globalización empresarial y del desarrollo de su aplicación que apoya con información oportuna y de calidad en favor de los gestores para la acertada toma de decisiones empresariales (Ramírez, et al., 2016).

Cabe resaltar que, Ecuador se encuentra incursionando en la difusión del lenguaje XBRL, es por esta razón que existen pocas instituciones que se están capacitando para su adopción, la mayor parte de empresas a nivel general transmiten información a las diferentes entidades de control en los formatos especificados por ellos, incluso existen compañías que se ven en la necesidad de transcribir los datos, porque los programas informáticos no permiten exportar directo esta información a un formato unificado (Laura, 2018), por lo que el intercambio de datos se da a través de archivos planos. Existen actores que se encuentran involucrados en el control y regulación de la SEPS, involucra la participación sectorial y transversal de las instituciones que se encuentran relacionadas con el sector. En la tabla 1, detalla la relación institucional de la SEPS. 


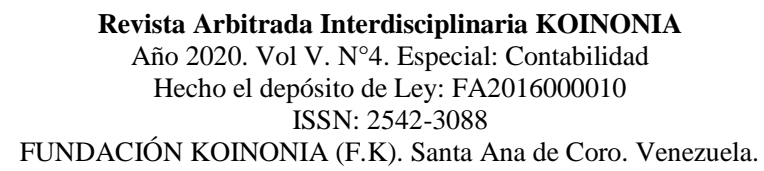

Edison Roberto Noblecilla-Ortiz; Edin Rodrigo Enriquez-Jaramillo; Verónica Paulina Moreno-Narváez

\section{MÉTODO}

La investigación fue de tipo no-experimental porque no se manipuló deliberadamente las variables, de tipo descriptiva-explicativa, puesto que se recopiló información de la taxonomía XBRL, economía popular y solidaria y precios justos, se describieron sus principales características en el referencial teórico, además tuvo un contexto explicativo, por cuanto a través del diagnóstico se obtuvo información relacionada con los costos de producción sobre los cuales se explicó su incidencia en la determinación de precios justos El diseño utilizado en el estudio fue transversal, por cuanto se levantaron datos en un solo momento con la participación de profesionales en el área de contabilidad y administración.

La técnica empleada para el levantamiento de la información fue la encuesta, la misma que se aplicó en 37 empresas dedicadas a la producción de bienes y servicios reguladas por la Ley de Economía Popular y Solidaria, los criterios para seleccionarlas estuvieron relacionados con la proximidad geográfica por la situación atravesada de la pandemia a nivel mundial y el conocimiento sobre costos de producción y lenguaje $X B R L$, analizada la información recopilada en función de la estadística descriptiva.

\section{RESULTADOS}

En esta etapa de la investigación se estableció el grado de conocimiento del lenguaje de reportes financieros XBRL para su aplicación e implantación a nivel de estructura de costos de producción, por tanto, los principales resultado se presentan a continuación:

\section{Conocimiento de la aplicación XBRL}

La estandarización de XBRL se puede aplicar en todos los países con normativas diferentes, sin embargo, se requiere de información y conocimiento en su taxonomía, es así que, en lo referente al nivel de conocimiento acerca de la terminología XBRL, el 49\% afirma no tener conocimientos sobre este lenguaje de programación, el 5\% consideraron tener altos conocimientos de la terminología, y el $30 \%$ de contadores consultados 
afirmaron tener un nivel de conocimiento bajo sobre esta temática (ver figura 4). En este sentido, se resalta la importancia de que los organismos de control efectúen procesos de capacitación y actualización de conocimientos con la finalidad de que la taxonomía XBRL sea conocida por los profesionales del área contable a nivel de la economía popular y solidaria.

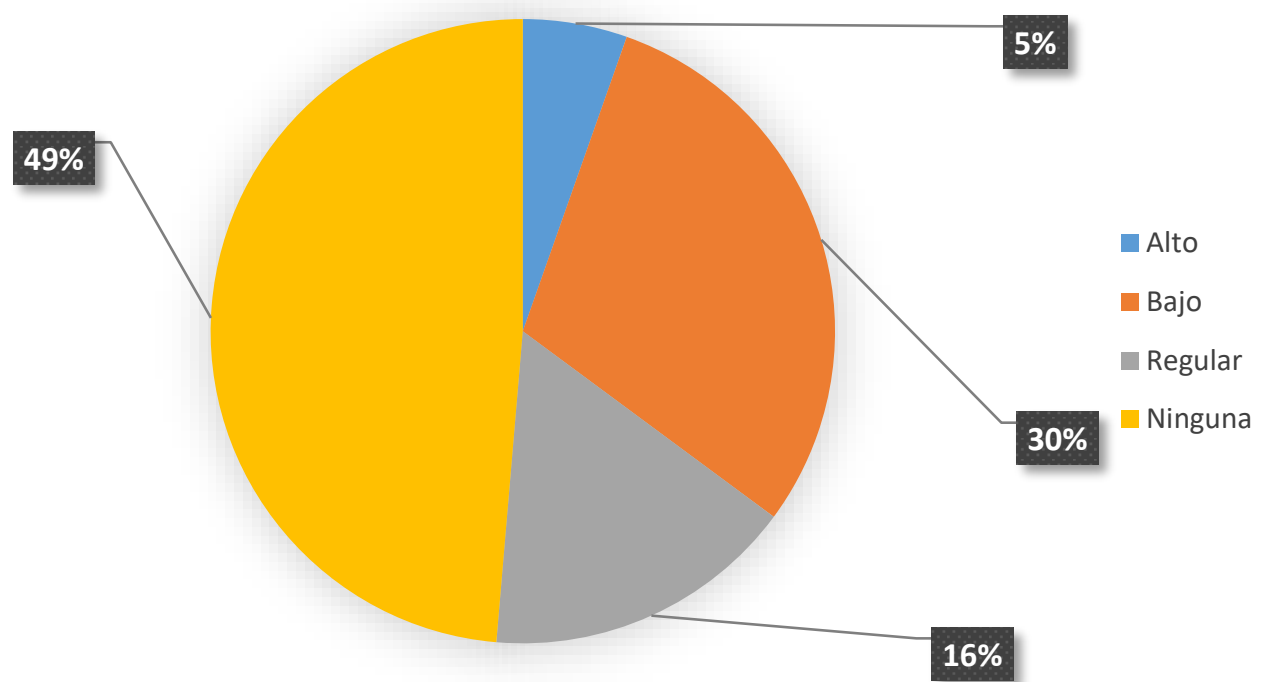

Figura 4. Conocimiento acerca de la terminología XBRL.

\section{Uso de tecnologías para la generación de información financiera}

La tecnología ha evolucionado de manera significativa, es por esto que, XBRL logra enviar información financiera y no financiera en un formato uniforme, claro y comparable, al respecto, los resultados reflejaron que en el $41 \%$ de empresas consultadas emiten estados financieros apoyados en un nivel alto por las herramientas tecnológicas, y tan solo un $13 \%$ mencionan que no cuentan con herramientas tecnológicas para la generación de estados financieros (ver figura 5). Los resultados dejan en evidencia que en el sector popular y solidario aun no se han consolidado las buenas prácticas de información financiera, pues la contabilidad es asumida como un requisito de los organismos de control y no como un insumo para la toma de decisiones de los gestores. 


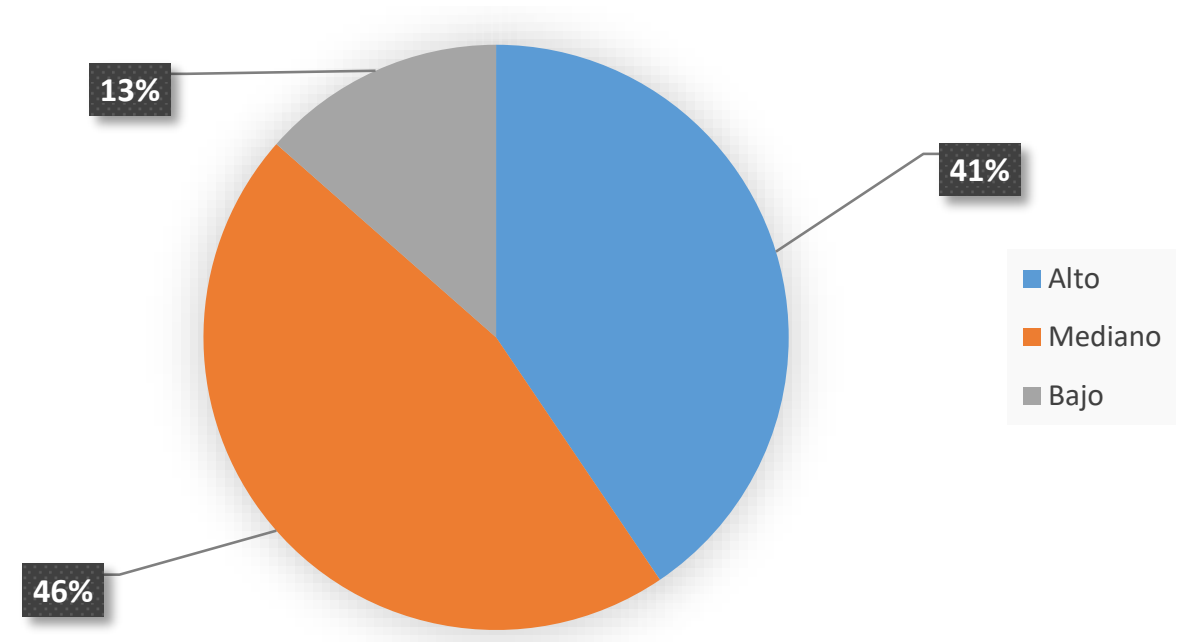

Figura 5. La influencia de la tecnología en la emisión de información financiera.

\section{Capacitación}

Es fundamental la capacitación de la aplicación XBRL, así como, su taxonomía y el alcance de su vinculación con los sistemas contables para la preparación de información financiera, por consiguiente, para el desarrollo del tema fue necesario consultar la opinión de los encuestados referente a su capacitación en la presentación de información financiera bajo la taxonomía NIIF/XBRL.

Al respecto, se determinó un alto grado de requerimiento de capacitación en estos temas por el $46 \%$ de profesionales encuestados, y apenas un $11 \%$ no lo consideran (ver figura 6 ), por lo tanto, los resultados obtenidos indican la necesidad de capacitar en temas relacionados con la adopción de las NIIF y la implementación del lenguaje $X B R L$, sin lugar a dudas, la necesidad de capacitación se da en este sector de la economía debido a que las empresas que se encuentran reguladas por la Superintendencia de Economía Popular y solidaria no tiene la obligación de adoptar las NIIF, en este sentido, los estados financiero siguen siendo elaborados bajo prácticas contables tradicionales. 
Revista Arbitrada Interdisciplinaria KOINONIA

Año 2020. Vol V. N². Especial: Contabilidad

Hecho el depósito de Ley: FA2016000010

ISSN: 2542-3088

FUNDACIÓN KOINONIA (F.K). Santa Ana de Coro. Venezuela.

Edison Roberto Noblecilla-Ortiz; Edin Rodrigo Enriquez-Jaramillo; Verónica Paulina Moreno-Narváez

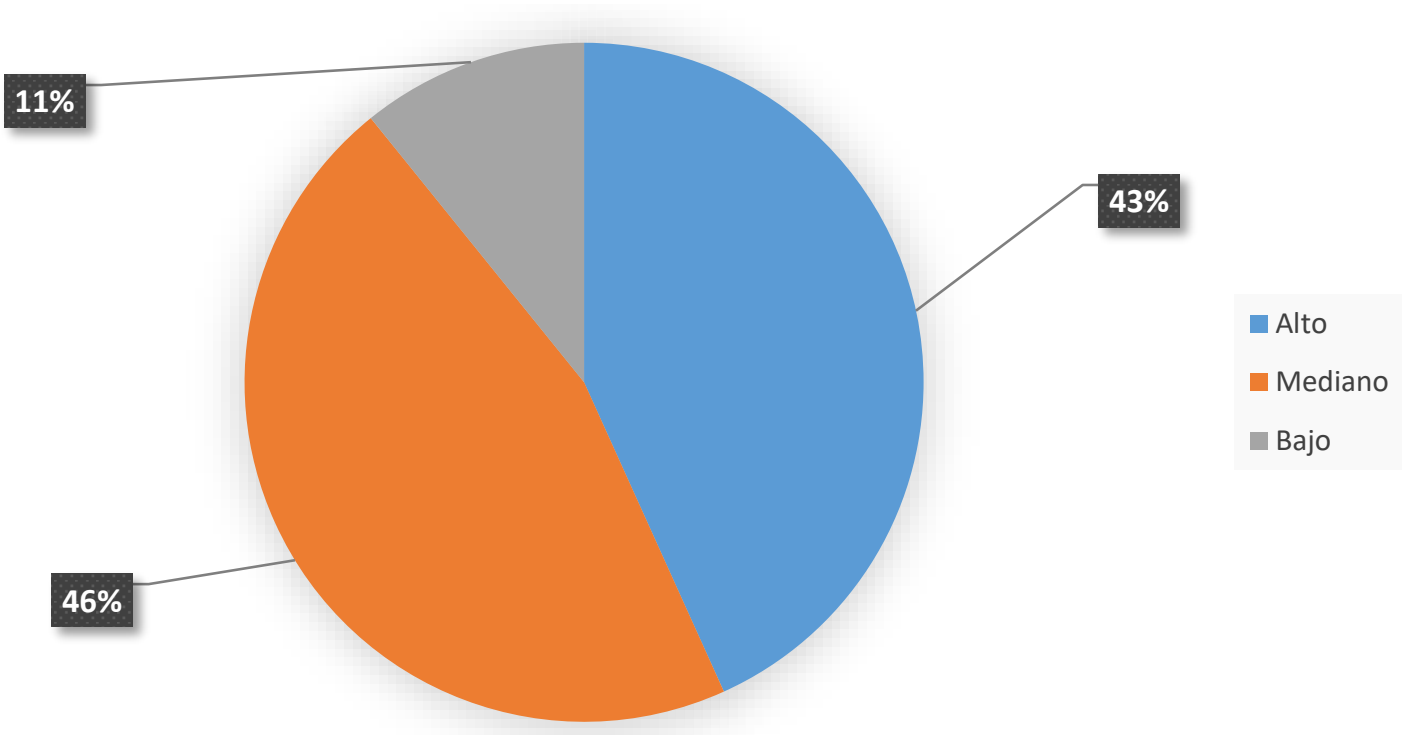

Figura 6. Requerimiento de capacitación en la taxonomía NIIF/XBRL. 


\section{PROPUESTA}

De los resultados obtenidos y basados en el grado de desarrollo internacional del XBRL, se considera necesario impulsar su utilización de forma inmediata con reglas y herramientas de desarrollo y validación propia, para prestar el mejor y moderno servicio a la sociedad ecuatoriana en transmisión de información económica-financiera bajo la regulación de una estructura de costos y precios justos. Por consiguiente, se propone un proyecto de implementación de XBRL por parte de la Superintendencia de Economía Popular y Solidaria para la generación de reportes de información financiera de las asociaciones, cooperativas o comunitarios dedicadas a la producción de bienes. La propuesta se sustenta en siete etapas que se encuentran identificadas en la figura 7 .

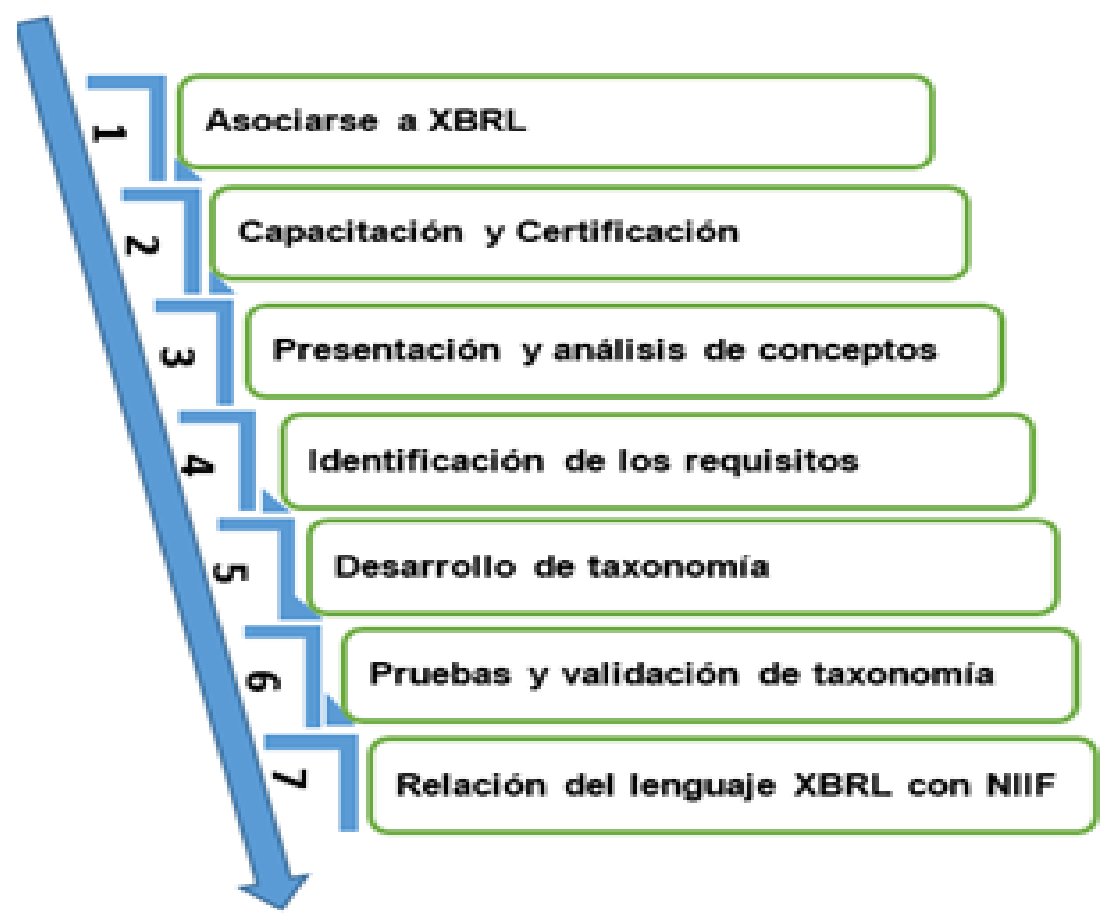

Figura 7. Estructura del proyecto de implementación de XBRL. 


\section{Asociarse a XBRL}

Un aspecto fundamental en el desarrollo del proyecto es contar con la Asociación obligatoria a XBRL Internacional mediante una jurisdicción XBRL. Las jurisdicciones son representaciones de países o regiones que tienen como objetivo promover el desarrollo y adopción del estándar XBRL a nivel local. Estas jurisdicciones posen los derechos exclusivos para representar a XBRL Internacional y tienen la capacidad de dar apoyo oficial a los proyectos e iniciativas de implementación XBRL. La Superintendencia de Economía Popular y Solidaria en el desarrollo de la implantación deberá tener claro que XBRL es un lenguaje para transmitir información bajo parámetros de exactitud y oportunidad.

\section{Capacitación y certificación}

La capacitación en XBRL en los principales aspectos financieros y tecnológicos, relacionados con en el desarrollo de las taxonomías y familiarización de los impactos de implantación del estándar XBRL para sistemas digitales de reportes corporativos debe llevarse a cabo por la Superintendencia de Economía Popular y Solidaria, quien deberá proveer el conocimiento adecuado para el entendimiento de los procesos de desarrollo de taxonomías. En la tabla 2 se establece las áreas de capacitación. 
Tabla 2.

Áreas de capacitación XBRL.

\section{Áreas de capacitación}

- Capacitación al personal contable de las cooperativas, asociaciones, comunitarios y entes de control.

- Adquisición de las herramientas necesarias para la creación de taxonomías y validación de informes XBRL.
- Implantación de ayudas técnicas para la creación de informes XBRL.

- Especificaciones, módulos de taxonomías, informes y reportes.

El objetivo principal de la capacitación es proporcionar una estructura de trabajo y de suministro de información de negocios para crear, cambiar y analizar la información de costos reportada, incluyendo la presentación de documentos reglamentarios como: estados financieros semestrales y anuales, información del libro mayor y programación de auditorías. De esta manera se logrará satisfacer la necesidad de contar con un lenguaje unificado en la economía popular y solidaria para generar información financiera con rapidez, confianza y consistencia relacionada con los costos de producción y determinación de precios justos.

\section{Presentación y análisis de conceptos}

En este punto se aplican pruebas de conceptos con alcance limitado, el objetivo es desarrollar las áreas de implantación y los beneficios potenciales del estándar XBRL. Esta prueba de conceptos permite examinar las condiciones reales para el proyecto de adopción de la taxonomía XBRL; como resultado del conocimiento práctico relacionado con la adopción para reportes específicos, este alcance permitirá evaluar los requerimientos reales y otorgará a los involucrados, habilidades y conocimientos para el desarrollo futuro en especificación de creación de variables como el tipo de moneda, idioma, periodo contable e ingreso de datos en la aplicación, coordinados por expertos en XBRL de la Superintendencia de Economía Popular y Solidaria. 


\section{Identificación de los requisitos}

Se debe identificar los requerimientos de contenidos XBRL, para ello, la Superintendencia de Economía Popular y Solidaria como órgano regulador deberá establecer como requisito que todas las asociaciones, cooperativas y comunitarios demuestren la capacitación en XBRL de su personal contable e informático para la implantación.

\section{Desarrollo de taxonomía}

La Superintendencia de Economía Popular y Solidaria en esta fase asumirá a las taxonomías como un conjunto de definiciones de un dominio de negocio, en este sentido, las especificaciones del lenguaje XBRL no definen por sí mismo conceptos de negocios, sino que establecen el lenguaje en el que se expresan. Las taxonomías definen los conceptos en un lenguaje formal. XBRL enuncia datos asociados a los conceptos definidos en una taxonomía para un contexto, o conjunto de contextos determinados. Un informe XBRL describe balances en diferentes periodos según los conceptos descritos. Las taxonomías se componen de esquemas y bases de datos relacionadas o link bases. En la figura 8, se observa de manera simplificada las acciones dadas una vez recibido el fichero XBRL.

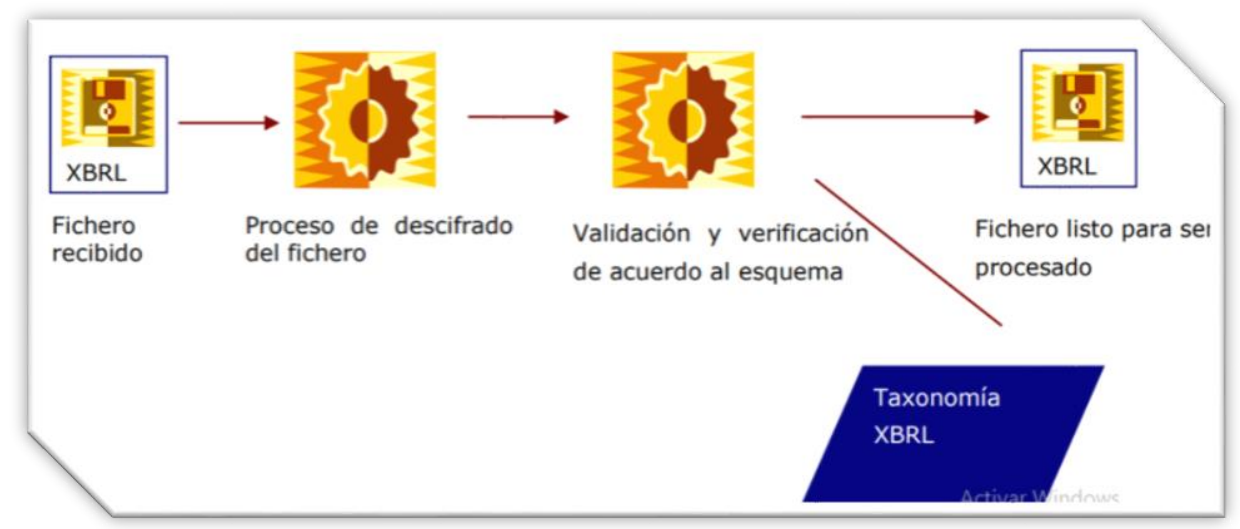

Figura 8. Extensión de taxonomías. 


\section{Pruebas y validación de taxonomías}

Las especificaciones generadas en esta etapa por $\mathrm{XBRL}$, deberá seguir un proceso de aprobación riguroso orientado a garantizar la calidad y estabilidad del material publicado. Se definirán pruebas y validaciones de la información financiera ingresada a la Superintendencia de Economía Popular y Solidaria a través de su página web:

1. Ingreso al sistema para enviar información.

2. Emisión de comprobante de ingreso de información.

3. Validación y envío de datos.

4. Notificación de éxito o fallo del envío de información a través del correo electrónico inscrito en la página de la SEPS.

Uno de los procesos fundamentales en el tratamiento de la información en XBRL es la validación de informes. El objetivo es asegurar la coherencia de la información reportada de acuerdo con las distintas reglas impuestas por el estándar y por el diseñador de la taxonomía.

\section{Relación del lenguaje XBRL con las NIIF}

Para alcanzar la transparencia de información financiera, la SEPS debe precisar la estructura de costos a fin de evitar la existencia de múltiples formatos que esconden o sobre valoran los costos y no permiten el establecimiento de precios justos. En la tabla 3 se precisan los componentes a considerar en la estructura de costos bajo el lenguaje XBRL y NIIF para PYMES 


\section{Tabla 3.}

Estructura de costos bajo el lenguaje XBRL.

\section{Estructura de costos mediante el uso de XBRL}

\begin{abstract}
Formato único para registrar los costos y gastos generados en el proceso productivo.

El sistema proporcionará el monto total de cada una de las cuentas de costos y gastos integrantes de la producción.
\end{abstract}

Se elaboraran estructuras de costos fijas

Se determinara el precio justo a partir del reconocimiento del valor menor entre los costos reales y estándar.

Se generará información clara y de calidad.

Se considerará los costos en función de las disposiciones de la NIIF para Pymes.

Los costos de producción se considerar tan solo como uno de los elementos de la información financiera

Sera responsabilidad de las cooperativas, asociaciones y comunitarios la integración de toda la información financiera en un solo sistema de información.
Las cooperativas, asociaciones y comunitarios deberán garantizar una arquitectura informática confiable.

Se excluirán de los costos de producción las cantidades excesivas de desperdicios.

El costo de producción estará relacionado con la materia prima, mano de obra y gastos indirectos de fabricación necesarios para la producción de bienes o servicios, según corresponda.

Se reconocerán los costos y gastos de producción solo si cuentan con la factura emitida por el vendedor.

Constituirán parte de los costos de adquisición el precio de las materias primas, los aranceles de importación, gastos de importación, transporte, manejo y almacenaje, así como otros costos atribuibles a la adquisición de materiales para la producción.

Se reconocerán los gastos de ventas, administración, representación y publicidad.

Se considerará el tratamiento de inventarios en correspondencia con las NIIF para Pymes.

Para la valoración de los inventarios se considerará el método de primeras entras , primeras salidas, y el método de promedio ponderado. 
Los estados financieros se elaborarán en apego a los principios de contabilidad generalmente aceptados.
Se reconocerán de manera exclusiva como parte de los costos los valores incurridos en condiciones de eficiencia normal.

\section{CONCLUSIÓN}

El lenguaje XBRL permitirá a las cooperativas, asociaciones y comunitarios del sector no financiero popular y solidario etiquetar datos financieros para procesar y compartir datos de manera eficiente, de esta forma se logrará generar información uniforme relacionada con los costos de producción y determinación de precios justos, así se crearán oportunidades para los productores menos favorecidos en condiciones dignas.

En Ecuador, los estados financieros son presentados por todas las empresas reguladas por los órganos de control, por esta razón, un aspecto prioritario en la implantación XBRL es pertenecer a una jurisdicción para el desarrollo de la taxonomía, mediante este proceso se podrá controlar el mercado por medio de los reportes financieros, para así obtener información que beneficie a todos los involucrados de manera particular a los pequeños productores.

Los resultados obtenidos a partir de la aplicación de la encuesta dejaron en evidencia que actualmente los negocios adscritos a la Superintendencia de Economía Popular y Solidaria elaboran sus estados financieros bajo las disposiciones de la Ley de Economía Popular y Solidaria y de la Ley Orgánica de Régimen Tributario Interno, sin embargo, su estructura adolece de un formato único, de una estructura fija, de la determinación exacta de los costos de producción y de un sistema de información eficiente que genere reportes de información financiera y no financiera útiles para la toma de decisiones.

Se identificó que existe un débil control en cuanto a la determinación de precios justos por parte de la Superintendencia de Control del Poder de Mercado, ya que las organizaciones que integran la economía popular y solidaria en su mayoría no determinan sus costos de manera adecuada, por tanto, la información generada de costos no tributa de manera eficiente a la determinación de precios justos. 


\section{FINANCIAMIENTO}

No monetario.

\section{AGRADECIMIENTO}

A la Universidad Católica de Cuenca; por impulsar el desarrollo de la investigación.

\section{REFERENCIAS CONSULTADAS}

Blacio-Aguilar, C., Narváez-Zurita, C., \& Erazo-Álvarez, J. (2020). Normas internacionales de información financiera y reconocimiento contable de jugadores de fútbol en clubes deportivos. [International standards for financial reporting and accounting recognition of soccer players in sports clubs]. Revista Arbitrada Interdisciplinaria Koinonía, 5(10), 34-62. doi:http://dx.doi.org/10.35381/r.k.v5i10.687

Cárdenas-Pañi, M., Narváez-Zurita, C., Erazo-Álvarez, J., \& Torres-Palacios, M. (2020). Conciliación de impuestos a las ganancias: Un estudio bajo la normativa fiscal y normativa contable. [Reconciliation of income taxes: A study under tax regulations and accounting regulations]. Revista Arbitrada Interdisciplinaria Koinonía, 5(10), 757-773. http://dx.doi.org/10.35381/r.k.v5i10.715

Laura, P. (2018). XBRL como cadena de abastecimiento de la información financiera. [XBRL as a supply chain for financial information]. Recuperado de https://bit.ly/2ZCF3A9

Leal, Y. (2015). ¿Es conveniente implementar XBRL en la transición a NIIF/IFRS? [ls it convenient to implement XBRL in the transition to IFRS / IFRS?]. Recuperado de https://bit.ly/3izRwNu

Lucero, A. (2017). Divulgación y apropiación del lenguaje XBRL por parte de las entidades públicas y de control de Colombia. [Divulgation and approval of XBRL in the part of the Community and the control of Colombia]. Recuperado de https://n9.cl/uzes

Lucero-Benítez, Á., \& Trujillo-Prada, M. (2018). Divulgación del lenguaje XBRL por parte de las entidades públicas y de control en Colombia [Disclosure of the XBRL language by public and control entities in Colombia]. Perspectivas, (9), 46-51.

Madrid, A., Cortés, J., \& Cardona, D. (2016). XBRL, ¿una realidad en Colombia? [XBRL, a reality in Colombia?]. Recuperado de https://bit.ly/3gvKurq 
Morán, E. (2014). Análisis de la institución pública de Control del Poder de Mercadoen El Ecuador frente a los órganos de control de la competencia en el derecho comparado. [Analysis of the public institution for the Control of Market Power in Ecuador compared to the compe]. Recuperado de https://bit.ly/3g6XV0e

Muñoz, M., Espinoza, R., Zúñiga, X., Guerrero, A., \& Campos, H. (2017). Contabilidad de costos para la gestión administrativa. [Cost accounting for administrative management]. Universidad Estatal de Milagro. Recuperado de https://bit.ly/348aoyy

Ordóñez-Granda, E., Narváez-Zurita, C., \& Erazo-Álvarez, J. (2020). El sistema financiero en Ecuador: Herramientas innovadoras y nuevos modelos de negocio [The financial system in Ecuador: Innovative tools and new business models]. Revista Arbitrada Interdisciplinaria Koinonía, 5(10), 195-225. doi:http://dx.doi.org/10.35381/r.k.v5i10.693

Ponte, E., Boixo, J., Rodríguez, T., \& Muñoz, F. (2007). XBRL, acercando las PYMES a los mercados del futuro. [XBRL, bringing pymes closer to the markets of the future]. Especial nuevas tecnologías, (194), 30-35.

Ramírez, M., Sepúlveda, M., \& Herrera, C. (2016). Lenguaje de los negocios: XBRL en España como referente para el manejo de la información contable y financiera en Colombia. [Business language: XBRL in Spain as a reference for the management of accounting and financial information in Colombia]. Obtenido de https://bit.ly/2NXkk4v

Ramírez, M., Sepúlveda-Cuervo, M. Y., \& Herrera-Camargo, C. J. (2016). Lenguaje de los negocios: XBRL en España como referente para el manejo de la información contable y financiera en Colombia. [Business language: XBRL in Spain as a benchmark for the management of accounting and financial information in Colombia]. In Vestigium Ire, 9(1), 87-104.

Superintendencia de Economía Popular y Solidaria. (2019). Plan estratégico 2019 - 2022 [Strategic plan 2019 - 2022]. Recuperado de https://bit.ly/3kTG40g

The Bussines Reporting Lenguage. (2020). ¿Qué es XBRL? [What is XBRL?]. Recuperado de https://bit.ly/3e4BcAZ

Zamarra, J., Atehortúa, T., \& Sierra, V. (2018). El XBRL: una herramienta hacia la comparabilidad de la información financiera [The XBRL: a tool towards comparability of financial information]. Recuperado de https://n9.cl/x9icc 
Revista Arbitrada Interdisciplinaria KOINONIA

Año 2020. Vol V. N 4 . Especial: Contabilidad

Hecho el depósito de Ley: FA2016000010

ISSN: 2542-3088

FUNDACIÓN KOINONIA (F.K). Santa Ana de Coro. Venezuela.

Edison Roberto Noblecilla-Ortiz; Edin Rodrigo Enriquez-Jaramillo; Verónica Paulina Moreno-Narváez

Zamarra, J.; Atehortúa, T., Sierra, V. (2017). Homogenización y comparabilidad de la información financiera a través del XBRL [Homogenization and comparability of financial information through the XBRL]. Contaduría Universidad de Antioquia, (71), 213-231

Zapata, P. (2019). Contabilidad de Costos. [Cost accounting]. Bogotá, Colombia. Alfaomega Colombiana S.A.

(C2020 por los autores. Este artículo es de acceso abierto y distribuido según los términos y condiciones de la licencia Creative Commons Atribución-NoComercial-Compartirlgual 4.0 Internacional (CC BY-NC-SA 4.0)

(https://creativecommons.org/licenses/by-nc-sa/4.0/ 